KINDER- UND JUGENDLITERATURFORSCHUNG 2002/2003 


\section{Kinder- und Jugendliteraturforschung 2002/2003}

\section{Mit einer Gesamtbibliographie}

der Veröffentlichungen des Jahres 2002

In Zusammenarbeit mit der Gesellschaft für Kinder- und

Jugendliteraturforschung in Deutschland und der deutschsprachigen Schweiz, der Österreichischen Gesellschaft für Kinder- und Jugendliteraturforschung

herausgegeben vom

Institut für Jugendbuchforschung der Johann Wolfgang Goethe-Universtität (Frankfurt am Main) und von der Kinder- und Jugendbuchabteilung der Staatsbibliothek Preußischer Kulturbesitz (Berlin)

unter der Verantwortung von

Bernd Dolle-Weinkauff, Hans-Heino Ewers und Carola Pohlmann

Wissenschaftlicher Beirat:

Andrea Immel (Princeton), Heinrich Kaulen (Hannover),

Ulrich Nassen (Leipzig), Bernhard Rank (Heidelberg), Karin Richter (Erfurt), Verena Rutschmann (Zürich), Barbara Scharioth (München), Ernst Seibert (Wien), Rüdiger Steinlein (Berlin), Gisela Wilkending (Köln) und Jack Zipes (Minneapolis)

\section{Verlag J. B. Metzler Stuttgart . Weimar}




\section{Redaktion: Bernd Dolle-Weinkauff \\ Rezensionen: Carola Pohlmann \\ Bibliographie: Karin Laubrecht \\ Layout: Antje Keil}

Der Druck dieses Jahrbuchs wurde durch die Gesellschaft für

Kinder- und Jugendliteraturforschung in Deutschland und

der deutschsprachigen Schweiz sowie die Deutsche

Forschungs-Gemeinschaft (DFG) gefördert.

Bibliografische Information Der Deutschen Bibliothek

Die Deutsche Bibliothek verzeichnet diese Publikation

in der Deutschen Nationalbibliografie; detaillierte bibliografische Daten

sind im Internet über <http $/ / d d b$.de $>$ abrufbar.

ISBN 978-3-476-01977-6

ISBN 978-3-476-02890-7 (eBook)

DOI $10.1007 / 978-3-476-02890-7$

Dieses Werk einschließlich aller seiner Teile ist urheberrechtlich geschützt. Jede Verwertung außerhalb der engen Grenzen des Urheberrechtsgesetzes ist ohne Zustimmung des Verlages unzulässig und strafbar. Das gilt insbesondere für Vervielfältigungen, Übersetzungen, Mikroverfilmungen und die Einspeicherung und Verarbeitung in elektronischen Systemen.

(C) 2003 Springer-Verlag GmbH Deutschland

Ursprünglich erschienen bei J. B. Metzlersche Verlagsbuchhandlung und Carl Ernst Poeschel

Verlag GmbH in Stuttgart 2003

www.metzlerverlag.de

info@metzlerverlag.de 


\section{Inhalt}

\section{Berichte}

Svenja Blume/Angelika Nix: Die Freiburger Arbeitsgruppe zur Kinder- und Jugendliteraturforschung in der Skandinavistik (FAKS) ...3.-Verena Rutschmann: Neuordnung der schweizerischen Kinderliteratur-Vermittlung: Das Schweizerische Institut für Kinder- und Jugendmedien...5

\section{Beiträge}

\section{Bettina Kümmerling-Meibauer}

(De)Kanonisierungsprozesse. Die Darstellung der Kinderliteratur in der Literaturgeschichtsschreibung vom Ende des 18. Jahrhunderts bis zur Gegenwart

Regina Wöllfert-Stockmann

Auf den Spuren der Schöpferin der Kleinen Dott.

Untersuchung zu Leben und Werk Tamara Ramsays.

Angelika Nix

Die Destruktion der poetischen Kindheit. Peter Pohls Roman

„Jan, mein Freund“ im Kontext der schwedischen Phantastik für Kinder.

\section{Tatjana Jesch}

Die alten Leiden der neuen Homunculi.

Über das Verhältnis zwischen Literatur und Philosophie

am Beispiel von Jugendromanen zum Thema Gentechnik

\section{Forschungsberichte}

Martin-Christoph Just

Ignorieren als literaturkritische Strategie.

Enid Blyton im Spiegel von Literatur-Lexika.

Rezensionen 
Die kontinuierliche Herausgabe eines Jahrbuches mit umfangreichem Rezensionsteil und einer umfassenden und dazu noch vergleichsweise aktuellen Fachbibliographie setzt den permanenten Einsatz von Lehrstuhlmitteln bzw. von Instituten und bibliothekarischen Einrichtungen voraus. Bereits in der Vergangenheit zeigte sich, dass sich nicht alle der bisherigen Herausgeber an der Editionsarbeit, wie eigentlich gewünscht, beteiligen konnten. Ausschlaggebend dafür waren neben den üblichen Lehr- und Forschungsbelastungen nicht zuletzt auch schmale Lehrstuhlausstattungen. Infolgedessen verlagerte sich die bibliographische und editorische Arbeit in zunehmendem Maße nach Frankfurt an das Institut und die Bibliothek für Jugendbuchforschung, in jüngster Zeit auch nach Berlin an die Kinderbuchabteilung der Staatsbibliothek Preußischer Kulturbesitz.

Beide Institutionen sind bereit, die editorischen Arbeiten bis hin zur Erstellung des Umbruchs zu tragen. Um die hierfür nötigen Kräfte und Mittel auch einsetzen zu können, ist es unerlässlich, die Herausgabe dieses Jahrbuches in den Kreis der offiziellen Aufgaben dieser Institutionen aufzunehmen. So bestand letztlich keine andere Wahl, als die Herausgeberschaft auf diese beiden Institutionen zu übertragen. Dabei zeichnen für das Institut und die Bibliothek in Frankfurt Bernd Dolle-Weinkauff und Hans-Heino Ewers, für die Berliner Kinderbuchabteilung Carola Pohlmann verantwortlich. Die bisherigen Herausgeber bleiben dem Jahrbuch als Mitglieder des wissenschaftlichen Beirats verbunden, dem auch die bisherigen Verbindungsherausgeber und einige weitere Persönlichkeiten angehören.

Für die vorliegende neunte Ausgabe von Kinder- und Jugendliteraturforschung wurde eine so große Zahl von Besprechungen eingereicht, dass diese den Umfang des Bandes zu sprengen drohten. Die Redaktion hat sich entscheiden müssen, einige Besprechungen zu kürzen und darüber hinaus den Platz für Beiträge und Forschungsberichte kleiner als in früheren Jahren zu halten. Auch wurde der Übersichtlichkeit halber eine neue Rubrik eingeführt, die Kurzrezensionen bietet. Dennoch mussten einige der eingesandten Rezensionen für die nächste Ausgabe zurückgestellt werden. Wir bitten um Verständnis für dieses Vorgehen, da der Umfang des Rezensionsteils in der Ausgabe 2002/2003 beinahe das Doppelte des Üblichen beträgt, und der Gesamtumfang des Bandes nicht beliebig vergrößert werden kann.

Wie bereits in den vergangenen Jahren versendet die Redaktion des Jahrbuchs an alle interessierten Bezieher kostenlos eine für IBM-kompatible PC-Systeme (MS-DOS/Windows) ausgelegte Datenbankversion der bisher veröffentlichten Jahresbibliographien (siehe Gutschein am Ende des Bandes). Da der Umfang der Bibliographie die Kapazität einer 1,44“-Diskette übersteigt, werden seit der Ausgabe 1999/2000 CD-ROM an die Bezieher verteilt.

Anschriften der Herausgeber:

Dr. Bernd Dolle-Weinkauff, Institut für Jugendbuchforschung der Johann Wolfgang GoetheUniversität, Postfach 111932, 60054 Frankfurt/M.

Prof. Dr. Hans-Heino Ewers, Institut für Jugendbuchforschung der Johann Wolfgang GoetheUniversität, Postfach 111932, 60054 Frankfurt/M.

Carola Pohlmann, Kinder- und Jugendbuchabteilung der Staatsbibliothek zu Berlin, 10102 Berlin 